\title{
Estructura de edades de pumas (Puma concolor) cazados bajo el sistema de remoción por recompensas en Patagonia. ¿Selectividad u oportunismo en la captura?
}

\author{
Romina Llanos $^{\bowtie, 1}$; Alejandro Travaini ${ }^{2}$; Silvana Montanelli ${ }^{3}$ \& EnRiQue CRespo ${ }^{1}$
}

1. Centro Nacional Patagónico. CONICET, Universidad Nacional de la Patagonia San Juan Bosco, Argentina. 2. Centro de Investigaciones Puerto Deseado, Universidad Nacional de la Patagonia Austral. CONICET, Argentina. 3. Dirección de Fauna y Flora Silvestre. Subsecretaría de Ganadería y Agricultura. Ministerio de Desarrollo Territorial y Sectores Productivos, Argentina.

\begin{abstract}
Resumen. El control letal de carnívoros es una práctica común, pero su eficacia ha sido escasamente evaluada y es aún poco clara. En Patagonia, el puma (Puma concolor) es considerado depredador de ganado ovino y su caza control es incentivada desde algunos estados provinciales mediante el pago de recompensas económicas. Esta medida de manejo no contempla la fiscalización ni monitoreo de los individuos removidos. Nuestro objetivo fue determinar la estructura de edades y sexos de los pumas recompensados en las provincias de Santa Cruz y Chubut bajo este sistema de control. Examinamos 411 cráneos colectados en ambas provincias. La edad relativa se determinó mediante un patrón de desgaste dentario, establecido en base a cráneos de edad conocida. El sexo fue especificado por los cazadores $u$ obtenido mediante un análisis discriminante basado en cuatro medidas craneales de especímenes de sexo conocido. La mayor proporción de individuos en ambas provincias corresponde a la clase de edad I o sub-adultos (entre 16 meses y dos años) (43.6\% Chubut y $46.5 \%$ Santa Cruz), seguidos de las crías (hasta 16 meses de edad) y las sucesivas clases de edad adultas. El sesgo hacia las clases más jóvenes podría deberse a una mayor abundancia de estos individuos en la población sumada a una mayor vulnerabilidad a la captura. La caza control por recompensas no es una medida que brinde soluciones reales al sector productivo. El esfuerzo y los recursos disponibles para el manejo del puma deberían destinarse al desarrollo de estrategias alternativas que contemplen los aspectos tratados en este trabajo y que redunden en beneficios tanto para el productor como para la conservación de la especie.
\end{abstract}

[Palabras clave: Manejo de depredadores, ganadería ovina, sistema de recompensas, distribución de edades]

\begin{abstract}
Age structure of cougars (Puma concolor) hunted under the bounty system in Patagonia. Selective or opportunistic capture?: The lethal control of carnivores in order to protect livestock is a common practice worldwide, but its effectiveness has been poorly evaluated and is still unclear. Pumas (Puma concolor) are considered by ranchers one of the main sheep predators in Patagonia, and their hunting is encouraged by the by the provincial estates through a state bounty system. This management measure does not include the examination and monitoring of the removed individuals. Our objective was to determine the age and sex structure of cougars hunted through the state bounty system in Chubut and Santa Cruz. We examined 411 skulls collected in both provinces. The age of each specimen was estimated by their tooth-wear pattern, established on the basis of skulls of known age. Sex was specified by hunters or obtained through a discriminant analysis based on four cranial measurements taken from specimens of known sex. The greatest proportion of individuals in both provinces corresponds to class I or sub-adults (between 16 months and two years of age) (43.6\% in Chubut and $46.5 \%$ in Santa Cruz), followed by cubs (up to 16 months old) and the successive age-classes. The bias towards younger age classes could be related to their relative abundance in the population together with their vulnerability to harvest. The state bounty system does not provide real benefits to livestock ranchers. Effort and resources for the management of cougars should be used to develop alternative strategies that address the issues discussed here and that benefit both the productive sector and the conservation of the species.
\end{abstract}

[Keywords: Predator management, sheep farming, state bounty system, age distribution]

\section{INTRODUCCIÓN}

El control letal de grandes carnívoros para proteger al ganado doméstico o a especies cinegéticas es una práctica extendida en todo el mundo (Knowlton et al. 1999; Treves \& Karanth 2003). El principal fundamento de esta medida subyace en el supuesto de que la sola eliminación de los predadores minimiza las tasas de depredación (Hone 1994). Así,

Editor asociado: Sergio Lambertucci

rllanos@cenpat-conicet.gob.ar los programas de control son aprobados o incluso incentivados desde el estado y varían de acuerdo al tipo de normativas, grado de selectividad y proporción de individuos removidos (Angst 2001; Woodroffe \& Frank 2005; Harper et al. 2007). A pesar de su extendida implementación, el efecto de estos programas sobre la actividad ganadera o las especies que se intentan favorecer ha sido escasamente evaluado (Berger 2006). 
Una estrategia de control frecuente es el pago de recompensas económicas por la caza de los predadores. En España, durante las décadas de 1950 y 1960, el estado promovió un sistema de recompensas por la captura y muerte de los llamados "animales dañinos", entre los que se incluían tanto aves rapaces como carnívoros (entre ellos el lince ibérico, Lynx pardinus, actualmente el felino más amenazado del mundo), para protección de especies cinegéticas (Corbelle \& Rico 2008). En Queensland (Australia), la depredación de ganado ovino por dingo (Canis lupus dingo) es un problema para la mayoría de los productores y el estado ha subsidiado la caza de este cánido durante muchos años y en diferentes niveles desde 1885 (Allen \& Sparkes 2001). En América del Norte, esta práctica también ha sido extendida por varias décadas para el control de coyotes (Canis latrans) y lobos (Canis lupus), considerados perjudiciales tanto para el ganado doméstico como para especies de valor cinegético (Omand 1950; West 1962; Bartel \& Brunson 2003; Hammill 2007).

El puma (Puma concolor, Linnaeus 1771), carnívoro con una amplia distribución en el continente americano (Currier 1983; Sunquist \& Sunquist 2002), fue extirpado de la mayor parte de su hábitat en América del Norte durante la segunda mitad del siglo XX, debido principalmente a los conflictos generados por la depredación sobre el ganado y especies cinegéticas (Anderson et al. 2010; Wainwright et al. 2010). La remoción casi total de este félido fue el resultado de campañas de erradicación masiva que se incentivaron por medio de remuneraciones económicas, pagadas tanto de manera privada como desde los estados. Estas medidas, fueron luego sustituidas por programas de manejo con restricciones sobre el sexo, edad y número de individuos que podían ser cazados. Bajo estas nuevas condiciones, a partir de 1990 la especie comenzó a recuperarse y restablecerse nuevamente en la región (Anderson et al. 2010).

En las provincias patagónicas del Chubut y Santa Cruz, Argentina, extremo sur de la distribución del puma, la depredación de ganado ovino es una de las principales causas de persecución de la especie (Walker y Norvaro 2010). El conflicto con el sector ganadero ha incrementado en las últimas décadas, debido fundamentalmente al restablecimiento del félido luego de una importante disminución en la actividad ganadera, que tuvo lugar a partir de la segunda mitad del siglo XX (Travaini et al. 2000; Walker \& Novaro 2010).
La reducción del número de ovejas comenzó con el deterioro de las pasturas, producto del sobrepastoreo (Golluscio et al. 1998; Aguiar \& Sala 1998, 1999). A esto se sumaron factores como la caída de los precios internacionales de la lana, la disminución de las ventas de carne ovina al exterior, la erupción del volcán Hudson en 1991 en Santa Cruz y la falta de inversión en recursos humanos e infraestructura, que agravaron la crisis de la ganadería ovina como actividad rentable (Andrade 2005; García Brea et al. 2010). En consecuencia, varios establecimientos rurales fueron cerrados y desprovistos de ovinos, lo que generó un patrón espacial conformado por extensiones de tierras abandonadas y despobladas entremezcladas a modo de mosaico con campos en producción (Barbería 1995; Bibiloni 2004; Andrade 2005, 2010; Llanos, obs. pers.). En este contexto, se desconoce el perjuicio económico real causado por el puma. La visión negativa que los productores tienen sobre la especie puede no ser objetiva, lo que genera una actitud hostil hacia su presencia en los campos y una fuerte presión sobre los agentes estatales para que se reduzcan e incluso eliminen sus poblaciones silvestres.

En respuesta a los reclamos del sector ganadero, en ambas provincias se declara al puma especie perjudicial para la ganadería y se recompensa su captura y muerte, actualmente con estipendio de \$500 (76 USD enero 2014) por individuo. En Santa Cruz, se permite la caza control de la especie y se paga cualquier individuo cazado, sin restricciones de ningún tipo, luego de la entrega de sus cueros. En Chubut, la Ley XVII 52 (ex ley 4100) de Control de Especies Depredadoras de la Ganadería establece un fondo impositivo a los productores, de acuerdo a la venta de lana, con el que se solventan los pagos de las recompensas que son efectivizadas mediante la entrega del cuero y cráneo del animal cazado. Desde mediados de 2009, la Dirección de Fauna y Flora Silvestre de la Provincia del Chubut sólo permite la caza selectiva del puma "problema" y autoriza el pago únicamente del animal que produce ataques al ganado, previa denuncia del productor rural. No obstante esta normativa, se carece aún de un método de fiscalización completo, por lo que en la práctica el sistema de pagos es en cierta medida similar al aplicado en Santa Cruz. Además de las políticas implementadas en ambas provincias, no existe aún un Plan de Manejo Regional ni de medidas alternativas destinadas a mitigar el conflicto y garantizar la conservación de la especie. 
Las características de la fracción de la población que ha sido removida por caza control indiscriminada es aún desconocida para ambas provincias. Sin restricciones de ningún tipo (en cuanto al rango etario, género o número de individuos que pueden ser recompensados) y en ausencia de un método de fiscalización de los ejemplares pagados, es esperable que las clases de edad y sexo estén representadas en las muestras en función de su disponibilidad y vulnerabilidad a la captura. Nuestros objetivos fueron determinar y comparar la estructura de edades y proporción de sexos de los pumas cazados en las provincias del Chubut y Santa Cruz en el marco del sistema de control por recompensas.

\section{MÉTOdos}

Los cráneos de los pumas cazados y remunerados en Santa Cruz, entre los años 1998 y 2011, fueron recolectados a través de las Sociedades Rurales, el Consejo Agrario Provincial y productores de esta provincia. Fueron trasladados, incorporados y examinados en la colección del Centro de Investigaciones Puerto Deseado (Universidad Nacional de la Patagonia Austral). Siempre que fue posible, se recabó información acerca de la edad, el sexo, el método de captura utilizado y la procedencia de cada individuo.

En Chubut, los cráneos de los pumas cazados entre los años 2005 y 2010 fueron entregados por los productores a las Sociedades Rurales (entidades encargadas de realizar el pago de la recompensa), almacenados transitoriamente en la Dirección Provincial de Flora y Fauna Silvestre de la provincia y finalmente trasladados al Centro Nacional Patagónico (CENPAT-CONICET). Para el cobro de cada espécimen se debió informar el sexo, edad relativa (cría, juvenil y adulto), método de caza (trampa, con perros, arma de fuego o veneno), fecha y lugar de captura y los cueros deben entregarse completos, con las cuatro garras y todas las uñas para evitar su comercialización.

Los individuos fueron clasificados en seis clases de edad en base al patrón de desgaste y coloración dentario, desarrollado por Anderson (1983) y descrito e ilustrado en Gay \& Best (1996a) y en Cougar Management Guidelines Working Group (2007). Pusimos a prueba esta clasificación con una muestra de 49 cráneos, cuyas edades absolutas estimamos a partir del recuento de bandas de crecimiento en cortes histológicos de la raíz de los dientes incisivos o caninos, donde cada banda corresponde a un año de vida (Klevezal \& Kleinenberg 1967; Crespo et al. 1994). Las clases de edad en base al desarrollo, desgaste y coloración de la dentadura general fueron seis (Tabla 1).

El sexo fue precisado por el cazador al momento de entregar la piel y el cráneo del animal cobrado, y se asume que esta clasificación es correcta dado que la condición sexual en los pumas se distingue por características externas, excepto en las crías donde no es tan evidente (Logan \& Sweanor 2001; Cougar Management Guidelines Working Group 2007). Para los especímenes que no contaban con esta información o resultó dudosa, la asignación del sexo se realizó mediante una función discriminante, computada en base a cuatro medidas craneales tomadas en individuos de sexo conocido y mayores a 16 meses (con todas las piezas dentarias totalmente erupcionadas). Estas medidas, que difieren significativamente entre machos y hembras en las diferentes edades (Gay \& Best 1995; Gay \& Best 1996a), fueron: (1) Largo Total (LT), largo medido desde el punto medio entre los incisivos superiores hasta el punto medio en el borde posterior del foramen magnum. (2) Largo del Paladar (LP), medido desde la extensión posterior del puente interpterigoideo, borde posterior, hasta la proyección anterior de los premaxilares. (3) Diámetro del Canino Superior (DCS), medido en la unión del cemento y el esmalte. (4) Profundidad

Tabla 1. Clases de edad obtenidas en base al desarrollo, desgaste y coloración de dientes de puma (Cougar Management Guidelines Working Group 2007).

Table 1. Age classes of cougar based on growth, wear and staining of the teeth.

\begin{tabular}{|c|c|c|c|}
\hline Clase de edad & Edad absoluta & Categoría & Características dentarias \\
\hline 0 & Hasta 16 meses & Crías & $\begin{array}{l}\text { Aún no se ha concluido el reemplazo total de los dientes deciduos } \\
\text { y la dentición definitiva no se encuentra totalmente erupcionada. }\end{array}$ \\
\hline I & $\begin{array}{l}\text { Entre } 16 \text { meses y dos } \\
\text { años }\end{array}$ & Sub-adultos & $\begin{array}{l}\text { Caninos e incisivos sin manchas de coloración amarillentas ni } \\
\text { desgaste. }\end{array}$ \\
\hline II & Tres y cuatro años & Adultos & $\begin{array}{l}\text { Caninos levemente manchados y con un ligero desgaste. El segundo } \\
\text { y tercer incisivo con ligero desgaste, el primer incisivo sin manchas } \\
\text { ni desgaste. }\end{array}$ \\
\hline III & Cinco y seis años & Adultos & $\begin{array}{l}\text { Caninos moderadamente manchados y con visible desgaste. El } \\
\text { primer y segundo incisivo con ligero desgaste. El tercer incisivo } \\
\text { con moderado desgaste. }\end{array}$ \\
\hline IV & $\begin{array}{l}\text { Siete, ocho y nueve } \\
\text { años }\end{array}$ & Adultos & $\begin{array}{l}\text { Caninos fuertemente manchados y con las cúspides redondeadas } \\
\text { o planas. El tercer incisivo desgastado hasta la altura del primer y } \\
\text { segundo incisivo. }\end{array}$ \\
\hline V & Mayores de 10 años & Adultos & $\begin{array}{l}\text { Los caninos fuertemente manchados y totalmente desgastados, } \\
\text { redondeados o romos. Incisivos fuertemente desgastados hasta la } \\
\text { línea de la encía o ausentes. }\end{array}$ \\
\hline
\end{tabular}


de la Mandíbula (PM), longitud desde el punto más distal de la mandíbula inferior hasta el punto medio más bajo en el borde inferior externo de la mandíbula.

Para este análisis, las clases de edad I y II se consideraron cada una por separado, mientras que las clases de edad III, IV y V fueron reunidas en un mismo grupo, ya que durante estas edades el crecimiento del cráneo cesa (Gay \& Best 1996a; Gay \& Best 1996b).

Por otro lado, y debido a que la función discriminante no puede aplicarse a individuos en los cuales alguna de las medidas no pudo ser tomada (por rotura o fragmentación del cráneo), se realizaron varios análisis según se describe en Gay \& Best (1996b). En el primero se incluyeron todos los individuos con datos completos. En los análisis subsecuentes una de las cuatro variables fue eliminada del set de datos para poder incluir a los especímenes con ese dato incompleto. Los pumas en los que faltaron dos o más medidas, no fueron incluidos en el análisis y se consideraron de sexo indeterminado.

Se evaluó si existen diferencias significativas en la proporción de individuos en cada clase de edad para ambas provincias, mediante un test de Gauss para diferencias de proporciones. Para evaluar si la relación sexual en cada clase de edad y para cada provincia fue diferente de 1:1 se utilizó la prueba de chi-cuadrado. En ambos análisis se supuso un nivel de significación de $5 \%(P<0.05)$. Para contrastar la estructura de edades de los pumas cazados en ambas provincias con las documentadas en la literatura para otras regiones, las clases de edad se agruparon luego como: crías (menores a los 16 meses de edad), sub-adultos (clase de edad I, individuos entre 16 meses y dos años) y adultos (individuos mayores a los tres años de edad; clase de edad II, III, IV y V).

\section{Resultados}

En total se examinaron 411 cráneos, de los cuales 211 pertenecieron a pumas procedentes del Chubut y 200 de Santa Cruz (Tabla 2). Los productores rurales especificaron el sexo de 148 cráneos (Tabla 3). Para los individuos de sexo conocido, el análisis discriminante asignó correctamente el sexo al 95\% de las hembras y el $91.7 \%$ de los machos de la clase de edad I; al 93.3\% de las hembras y el 100\% de los machos de la clase de edad II y al 100\% de las hembras y al $80 \%$ de los machos en las clases de edad III- IV-V. Esto permitió realizar de manera confiable la clasificación de los individuos mayores a los 16 meses de edad cuya condición sexual era desconocida.

No se encontraron diferencias significativas en la proporción de individuos en cada clase de edad entre Chubut y Santa Cruz $(P>$
Tabla 2. Número de cráneos de pumas colectados por año en las provincias del Chubut y Santa Cruz, Argentina, bajo el sistema de control por recompensas vigente en ambas provincias.

Table 2. Number of cougar skulls collected annually under the state bounty system in Chubut and Santa Cruz provinces, Argentina.

\begin{tabular}{ccc}
\hline Año & Chubut & Santa Cruz \\
\hline 1998 & - & 4 \\
2004 & - & 2 \\
2005 & 2 & 38 \\
2006 & 83 & 39 \\
2007 & 52 & 14 \\
2008 & 30 & - \\
2009 & 22 & 27 \\
2010 & 2 & - \\
2011 & - & 32 \\
Desconocido & 20 & 44 \\
Total & 211 & 200 \\
\hline
\end{tabular}

0.05, según Test de Gauss para diferencias de proporciones) (Tabla 4). Las estructuras de edades en ambas provincias estuvieron compuestas en su mayor parte por individuos de clase de edad I (sub-adultos, dos años), seguidos por los de clase de edad 0 (crías, hasta 16 meses de edad) y los de la clase de edad II (adultos de entre tres y cuatro años) (Tabla 4). Los adultos de las clases de edad III (cinco, seis y siete años), IV (ocho y nueve años) y V (mayores a los diez años) fueron los menos representados, constituyendo alrededor del $10 \%$ del total en cada muestra.

La proporción de sexos para cada clase de edad difirió entre las provincias estudiadas (Tabla 5). En Chubut, el número de individuos identificados como machos en la clase de edad 0 fue mayor al de hembras (proporción sexual 1.9 Machos: 1 Hembras; $X^{2}=4.26$; g.l. $=1 ; P<$ 0.05), en Santa Cruz sólo uno de los cráneos contaba con información del sexo por lo que no se pudo establecer la proporción sexual en este

Tabla 3. Número de cráneos clasificados por clase de edad y sexo, provenientes de pumas cazados en las provincias del Chubut y Santa Cruz, Argentina.

Table 3. Number of skulls classified by age classes and sex, of cougars hunted in Chubut and Santa Cruz provinces, Argentina.

\begin{tabular}{ccccc}
\hline $\begin{array}{c}\text { Clases de } \\
\text { edad }\end{array}$ & $\begin{array}{c}\mathrm{N}^{\circ} \text {. } \\
\text { Hembras }\end{array}$ & $\begin{array}{c}\mathrm{N}^{\circ} \text {. } \\
\text { Machos }\end{array}$ & $\begin{array}{c}\text { Sexo No } \\
\text { identificado }\end{array}$ & Total \\
\hline 0 & 17 & 30 & 68 & 115 \\
I & 26 & 33 & 126 & 185 \\
II & 17 & 10 & 44 & 71 \\
III & 5 & 2 & 16 & 23 \\
IV & 1 & 5 & 4 & 10 \\
V & 0 & 2 & 5 & 7 \\
Total & 66 & 82 & 263 & 411 \\
\hline
\end{tabular}


Tabla 4. Estructura de edades y sexo de las muestras de pumas capturados bajo la modalidad de caza control por recompensas en las provincias del Chubut y Santa Cruz, Argentina.

Table 4. Age and sex structure of cougars hunted under the state bounty system in Chubut and Santa Cruz provinces, Argentina.

\begin{tabular}{ccccc}
$\begin{array}{c}\text { Clase de } \\
\text { edad }\end{array}$ & $\begin{array}{c}\text { Hembras } \\
\%(\mathrm{~N})\end{array}$ & $\begin{array}{c}\text { Machos } \\
\%(\mathrm{~N})\end{array}$ & $\begin{array}{c}\text { Indeterminado } \\
\%(\mathrm{~N})\end{array}$ & $\begin{array}{c}\text { Total } \\
\%(\mathrm{~N})\end{array}$ \\
\hline \multicolumn{5}{c}{ Chubut } \\
0 & $7.58(16)$ & $14.22(30)$ & $7.11(15)$ & $28.91(61)$ \\
I & $23.22(49)$ & $19.91(42)$ & $0.47(1)$ & $43.60(92)$ \\
II & $11.85(25)$ & $5.69(12)$ & 0 & $17.54(37)$ \\
III & $3.32(7)$ & $2.84(6)$ & 0 & $6.16(13)$ \\
IV & $1.42(3)$ & $1.42(3)$ & 0 & $2.84(6)$ \\
V & $0.95(2)$ & $0(0)$ & 0 & $0.95(2)$ \\
Total / \% $48.34(102)$ & $44.08(93)$ & $7.58(16)$ & 211 \\
(N) & \multicolumn{5}{c}{ Santa Cruz } \\
0 & $0.5(1)$ & 0 & $26.5(53)$ & $27(54)$ \\
I & $15(30)$ & $31(62)$ & $0.5(1)$ & $46.5(93)$ \\
II & $5(10)$ & $12(24)$ & 0 & $17(34)$ \\
III & $3.5(7)$ & $1.5(3)$ & 0 & $5(10)$ \\
IV & $0.5(1)$ & $1.5(3)$ & 0 & $2(4)$ \\
V & $1.5(3)$ & $1(2)$ & 0 & $2.5(5)$ \\
Total / \% & $26(52)$ & $47(94)$ & $27(54)$ & 200 \\
(N) & & & & \\
\hline \multicolumn{5}{c}{} \\
\end{tabular}

rango etario. En la clase de edad I, la relación de sexos no difirió de 1:1 en Chubut $\left(\mathrm{X}^{2}=\right.$ 0.54; g.l. $=1 ; P>0.05)$, aunque en Santa Cruz estuvo sesgada hacia los machos (proporción sexual 2.06 M:1 H; $\mathrm{X}^{2}=11.13$; g.l. $=1 ; P<0.05$ ). En la clase de edad II las hembras estuvieron mayormente representadas que los machos en Chubut (proporción sexual $1 \mathrm{M}: 2.3 \mathrm{H}$; $\mathrm{X}^{2}=4.57$; g.l. $\left.=1 ; P<0.05\right)$, pero la relación se invirtió en Santa Cruz (proporción sexual 2.4 $\mathrm{M}: 1 \mathrm{H} ; \mathrm{X}^{2}=5.76 ;$ g.l. $\left.=1 ; P<0.05\right)$. El pequeño tamaño de muestra para las clases de edad III, IV y V no permitió evaluar las diferencias en la proporción de sexos para ninguna de las dos provincias.

Tabla 5. Porcentaje de machos y hembras para las clases de edad 0, I y II de Chubut y para las clases de edad I y II de Santa Cruz.

Table 5. Percentage of males and females belonging to age classes 0 , I and II of Chubut and age classes I and II of Santa Cruz.

\begin{tabular}{|c|c|c|}
\hline Clase de edad & \% Hembras & $\%$ Machos \\
\hline \multicolumn{3}{|c|}{ Chubut } \\
\hline 0 & 34.78 & 65.22 \\
\hline I & 53.85 & 46.15 \\
\hline II & 67.57 & 32.43 \\
\hline \multicolumn{3}{|c|}{ Santa Cruz } \\
\hline I & 32.61 & 67.39 \\
\hline II & 29.41 & 70.59 \\
\hline
\end{tabular}

El método de caza fue especificado por los productores rurales para 83 cráneos colectados en Chubut (39.34\% del total), para el resto de la muestra de esta provincia y la de Santa Cruz se desconoce la manera en que los especímenes fueron capturados. El más utilizado fue el trampeo (71\% de los casos), seguido de la persecución directa con perros y armas de fuego (21.7\%). En menor medida se utilizaron el veneno (3.6\%) y el guachi $(3.6 \%)$, que consiste en la colocación de un lazo de cuello en los lugares de paso del puma ya sea en un alambrado o en cualquier sitio que tenga las condiciones para instalarlo. En cuanto a la época del año en que los individuos fueron cazados, la información disponible para el $70 \%$ de los cráneos de Chubut indica que el $60 \%$ fue cazado en los meses de invierno y primavera.

\section{DisCUSIÓN}

La estructura de edades y sexo de los pumas removidos en Patagonia mediante el sistema de recompensas era hasta ahora desconocida. La probabilidad de que un sexo o clase de edad sea capturado está determinada por su abundancia relativa en la población y por su vulnerabilidad a la captura (Anderson \& Lindzey 2005). En una investigación realizada en Wyoming (EE.UU), en la que se redujo el tamaño de una población de pumas permitiéndose luego su recuperación, se observó que las clases de edad con mayor vulnerabilidad son capturadas hasta que se reduce su abundancia y comienza a extraerse el siguiente grupo más vulnerable (Anderson \& Lindzey 2005). Era esperable que la composición de las muestras obtenidas por caza en Chubut y Santa Cruz estuviera determinada por esos dos factores dado que, sin restricciones de ningún tipo y en ausencia de un método de fiscalización de la medida de manejo, se recompensó indiscriminadamente cualquier ejemplar capturado. Ambos factores pueden afectar la proporción de cada clase de edad y sexo, sumando sus efectos o de forma independiente si el peso de uno de ellos es muy significativo respecto del otro.

La estructura de edades en ambas provincias, con predominancia de individuos sub-adultos, fue diferente a la hallada para poblaciones estables no sometidas a explotación humana, compuestas principalmente de adultos, seguidos de las crías y por último los subadultos (Logan \& Sweanor 2001; Stoner et al. 2006). Nuestros resultados se asemejan a los obtenidos por Anderson \& Lindzey (2005) para 
una fracción de pumas removida por cacería, cuando los niveles de extracción fueron bajos y la población se encontró en una fase de recuperación o con una alta densidad. En este sentido, el sesgo hacia los sub-adultos en las muestras de Patagonia podría reflejar una población joven, en proceso de recuperación. El restablecimiento de la especie en la región ha sido asociado al decrecimiento de la actividad ganadera, que comenzó en las últimas décadas del siglo XX (Travaini et al. 2000; Walker \& Novaro 2010) y se desencadeno por factores climáticos y antrópicos que llevaron a la disminución del número de ovejas y de la población rural (Golluscio et al. 1998; Aguiar \& Sala 1998, 1999; Andrade 2005; García Brea et al. 2010). Esto hizo que se reduzca la presión de caza sobre especies que fueron intensamente perseguidas por años, como el guanaco (Lama guanicoe) y el puma, y comiencen a recuperarse posteriormente (Walker \& Novaro 2010).

La alta representación de los sub-adultos podría estar determinada además por su mayor vulnerabilidad a los métodos de caza. Los patrones de movimiento que exhiben los pumas, según la clase de edad y sexo al que pertenezcan, los exponen a distintos riegos de ser cazados (Barnhurst 1986). Estos individuos, especialmente los machos, en su etapa de dispersión recorren grandes distancias para establecerse en un nuevo territorio (Logan \& Sweanor 2001). Es durante esta recorrida que aumentaría la probabilidad de que sean trampeados o encontrados por el hombre de manera directa. La mayor vulnerabilidad durante el período de dispersión también ha sido observada en otras especies de félidos, como el lince (Lynx rufus) y el ocelote (Leopardus pardalis) (Kamler \& Gipson 2000; Haines et al. 2005). Además, la experiencia para evitar ser capturados aumenta conforme a la edad, como por ejemplo ocurre con los zorros rojos (Vulpes Vulpes) que llegados a la edad adulta han aprendido a evadir las trampas e incluso a como escapar de ellas, habilidad que los más jóvenes aún no tienen (Storm et al. 1976).

Las crías también estuvieron fuertemente representadas en ambas provincias, pero la proporción podría ser aún mayor dado que además de estar expuestos a la caza directa pueden morir en sus madrigueras por falta de alimento si su madre ha sido cazada. Camadas capturadas junto a las hembras han sido remuneradas individualmente, incluso en Santa Cruz se han recompensado individuos nonatos obtenidos de una hembra en gestación (Travaini, obs. pers.). En Chubut, para evitar recompensar individuos que aún no producen ataques al ganado, la DFyFS prohibió el pago de cueros cuyo largo total (medido desde el extremo del hocico al extremo de la cola) sea menor a 1.8 metros (Disposición N $\mathrm{N}^{\mathrm{0}}$ 9/2012 de 2012). Sin embargo, a partir de enero de 2013 se dejó sin efecto está disposición bajo la argumentación de que pumas cuyas medidas fueron menores al largo estipulado han depredado ovinos y muchos ejemplares no fueron entregados, perdiendo así información valiosa para los estudios. Actualmente en Chubut no se pagan las crías, si estas son capturadas junto a su madre sólo se remunera la hembra.

La relación de sexos de las crías fue estimada solo para Chubut y estuvo sesgada hacia los machos, contrariamente a lo hallado en poblaciones tanto silvestres como de cautiverio, donde la proporción sexual fue de 1:1 (Robinette et al. 1961; Seidensticker et al. 1973; Logan \& Sweanor 2001). El número de machos de nuestro estudio podría estar sobrestimado ya que a partir de las cuatro semanas de edad su genitalidad se puede distinguir con mayor facilidad (Cougar Management Guidelines Working Group 2007) y aquellos individuos no identificados (15 cráneos) podrían ser en su mayoría hembras que los cazadores no consiguieron clasificar.

Entre los adultos, la proporción sexual varió para cada provincia, con más hembras que machos en Chubut y contrariamente en Santa Cruz. No contamos con información suficiente ni con evidencias que permitan identificar los factores que podrían haber influido en la relación de sexos de esta clase de edad, pero podemos proponer algunos. Por ejemplo, una diferencia estacional en la vulnerabilidad a la captura, particularmente de las hembras, si la cacería se concentra en el período del año en el que se encuentran recluidas en sus madrigueras con las crías recién nacidas o en la época posterior de mayor actividad y movimiento. Los pulsos de nacimiento se dan principalmente en los meses de calor (Robinette et al. 1961; Ross \& Jalkotzy 1992; Logan \& Sweanor 2001; Ruth 2004), durante los cuales las hembras permanecen mayormente en sus madrigueras dedicadas al cuidado de sus camadas. En invierno suelen recorrer áreas mucho más grandes en busca de alimento tanto para ellas como para las crías que comienzan a destetar, aumentando el riesgo de ser capturadas (Knopff et al. 2010). La información disponible no nos permite 
realizar un análisis específico sobre esta posible causa del sesgo hacia las hembras en Chubut, pero los registros recabados indican que gran parte de los pumas fueron cazados en los meses de invierno y primavera, durante los cuales las hembras estarían más expuestas a los encuentros con el hombre. Asimismo, podría existir una vulnerabilidad diferencial entre machos y hembras a los métodos de caza. En especies de carnívoros como el zorro rojo (Vulpes vulpes), se ha observado que se cazan más machos que hembras con disparos de arma de fuego y, contrariamente, más hembras que machos por medio de trampas (Storm et al. 1976). En Chubut uno de los métodos mayormente utilizado fue el trampeo, lo que podría determinar el sesgo hacia las hembras. Además, existen otros factores a considerar como una asignación errónea del sexo o diferencias en la disposición espacial "campos en actividad-campos cerrados", esto último relacionado a la dinámica fuentesumidero que exhiben las poblaciones de pumas (Robinson et al. 2008).

En cuanto a los beneficios que se intentan obtener para la actividad ganadera, el sesgo en las capturas hacia las clases de edad más jóvenes no garantiza una disminución en el daño por depredación. Incluso, el perjuicio se podría ver aumentado localmente por el flujo de individuos que se promueve al dejar áreas vacantes en los territorios. En América del Norte, la eliminación de pumas en áreas pequeñas conlleva altos niveles de inmigración desde zonas vecinas, que actúan como fuentes procreadoras de individuos que se trasladan a esas áreas y las mantienen estables (Logan et al. 1986; Ross \& Jalkotzy 1992; Logan \& Sweanor 2001; Anderson \& Lindzey 2005; Stoner et al. 2006; Robinson et al. 2008). Para otras especies de carnívoros como el lobo (Canis lupus) y el zorro rojo (Vulpes vulpes) se documentaron rápidas repoblaciones en las zonas donde se aplican regímenes de control (Bjorge \& Gunson 1985; Reynolds et al. 1993). La alternancia de campos abandonados y campos en producción en Chubut y Santa Cruz conforma un escenario propicio para esta dinámica fuente-sumidero, donde la mortalidad por caza es compensada resultando en una baja reducción del número de pumas, inutilizando el esfuerzo y el dinero que se emplean para tal fin. En apoyo a esta conjetura, según los registros recabados en Chubut el número de pumas cazados no ha disminuido sino que tiende a aumentar (Figura 1). Además, suponemos que existe una cierta cantidad de especímenes que son removidos

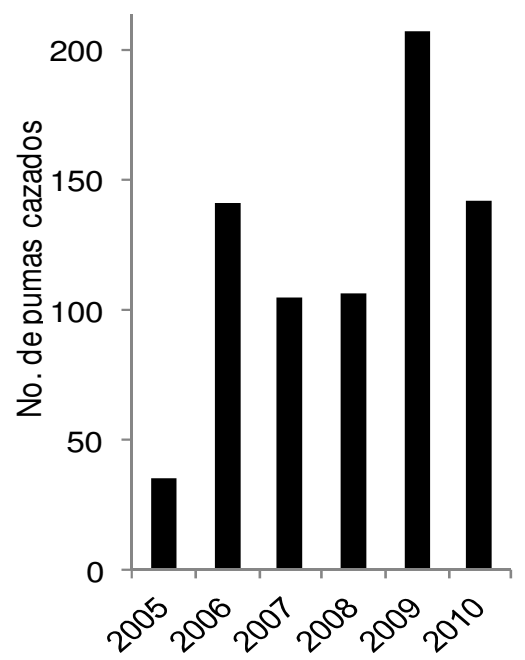

Figura 1. Número total de pumas cazados y cobrados bajo el sistema de control por recompensas implementado en la provincia del Chubut, entre los años 2005 y 2010.

Figure 1. Total number of cougars hunted and paid under the state bounty system in Chubut province, during the period 2005-2010.

pero no declarados, ya que en muchos casos no se entregan las pieles y cráneos de los ejemplares capturados. La pérdida de estos registros podría incorporar un sesgo en estudios de este tipo y si bien este método no es el mejor para estimar poblaciones, puede ser útil cuando no hay información sobre la especie.

Mediante la caza indiscriminada se dejan áreas vacantes que, teniendo en cuenta que no todos los pumas depredan sobre ganado (Elbroch \& Wittmer 2013), pueden ser ocupadas por individuos más propensos a realizar ataques de ovinos que el que fue removido. Una estrategia alternativa es la caza selectiva de los individuos "problema", es decir, aquellos que producen el daño. Esta medida puede ser aplicada si luego del evento de depredación se inspeccionan las carcasas frescas y los rastros hallados en el lugar, lo que permitirá rastrear e identificar al responsable del ataque. En EE.UU. se aplica este sistema y su funcionamiento involucra la cooperación tanto de los organismos estatales como de las personas interesadas en el manejo del puma y en la reducción de la depredación de ganado (Suminski 1982; Beausoleil \& Martorello 2005). Si bien requiere de inversión económica y de recursos humanos, podría ser una medida rentable en términos de costos/beneficios si se tiene en cuenta la reducción del daño que puede alcanzarse.

En Chubut se actualizó la legislación en 2009 para todo el territorio provincial y se autoriza 
sólo la caza selectiva del puma problema, previa denuncia de ataques al ganado (Montanelli 2008). Sin embargo, la carencia de recursos e infraestructura para corroborar la depredación e identificar de manera inequívoca al individuo responsable hacen que esta medida no se cumpla en forma efectiva y que sea en cierto grado similar a la practicada en Santa Cruz, donde se recompensan todos los pumas cazados. En Chubut desde el año 2010 se realizan reuniones con la Federación de las Sociedades Rurales y productores en las que se brinda información acerca de los resultados de los estudios realizados con el material obtenido de las capturas, con el objetivo de concientizar acerca de la caza selectiva.

Por último, un aspecto controversial del manejo del puma es la visión que el sector ganadero tiene sobre la especie, que puede estar sobredimensionada. Existen otros factores que ocasionan pérdidas de ovinos como la sequía, las nevadas, enfermedades y abigeato (Irazoqui 1981; Hall \& Paruelo 2006; Llanos, obs. pers.). Sin embrago, se consideran subjetivamente a los ataques por puma como una de las principales causas de pérdidas, ya que aún no hay información empírica que lo pruebe. Asimismo, la ausencia de prácticas ganaderas adecuadas y de inversiones en la infraestructura y recursos humanos de los campos son componentes importantes en detrimento de la actividad pecuaria (Brea et al. 2010) y tampoco son considerados como parte del problema. Sumado a esto no se han realizado evaluaciones sobre la frecuencia de ataques al ganado y, por lo tanto, se desconoce el efecto del control de la especie sobre la reducción de la depredación. En este contexto, la remoción indiscriminada de una especie nativa no es una solución a la crisis del sector productivo lanero y el pago de recompensas por pumas resulta en una compensación social, que tiene más la función de apaciguar los reclamos de los productores que de reducir las pérdidas de ganado ocasionadas por la especie. Por lo tanto, creemos que el esfuerzo y los recursos disponibles deberían destinarse al desarrollo de estrategias más efectivas que contemplen los aspectos aquí tratados y que redunden en beneficios tanto para el sector productivo como para la conservación de la especiey, en consecuencia, del ambiente donde se desarrollan las actividades humanas.

Agradecimientos: Agradecemos al Consejo Agrario Provincial de Santa Cruz y a la Dirección de Fauna y Flora Silvestre de Chubut, por la cooperación en la colecta, limpieza y almacenamiento del material de estudio. A las Sociedades Rurales de ambas provincias por la colaboración en la colecta del material. A CONICET y a la Universidad Nacional de la Patagonia Austral por su soporte financiero. A D Procopio por su asistencia en la clasificación de las muestras de Santa Cruz. A M Nabte, A Andrade y P Ferreras por la lectura crítica de este manuscrito y a los dos revisores anónimos que contribuyeron a mejorar la versión final.

\section{BIBLIOGRAFÍA}

AgUiAR, MR \& OE SALA. 1998. Interactions among grasses, shrubs, and herbivores in Patagonian grass-shrub steppes. Ecol. Austral, 8:201-210.

Aguiar, MR \& OE SALA. 1999. Patch structure, dynamics and implications for the functioning of arid ecosystems. Trends Ecol. Evol., 14:273-277.

AlLEN, LR \& EC SPARKES. 2001. The effect of dingo control on sheep and beef cattle in Queensland. J. Appl. Ecol., 38:76-87.

ANDERSON, AE. 1983. A critical review of literature on puma (Felis concolor). Special Report Number 54. Colorado Division of Wildlife (Wildlife Research Section). Fort Collins, Colorado, USA.

Anderson, CR \& FG Lindzey. 2005. Experimental evaluation of population trend and harvest composition in a Wyoming cougar population. Wildl. Soc. Bull., 33: 179-188.

Anderson, CR; FG LINDZEY; KH KNOPFF; MG JALKOtZy \& MS BOYCE. 2010. Cougar management in North America. Part I, Chapter 4, Pp. 41-54 en: M Hornocker y S Negri (eds.). Cougar, Ecology and Conservation. University of Chicago Press. Chicago, USA. 304 pp.

Andrade, L. 2005. Sociología de la desertificación. Los productores ovinos extensivos de la Patagonia Austral. Minio \& Dávila Editores. Buenos Aires. 388 pp.

ANDRADE, L. 2010. Otoño en la Estepa. Ambiente, Ganadería y Vinculos en la Patagonia Austral. Editorial La colmena. Buenos Aires. 306 pp.

AnGst, C. 2001. Procedure to Selectively Remove Stock Raiding Lynx in Switzerland. Carnivore Damage Prevention News, 4:8.

Barbería, E.M. 1995. Los dueños de la tierra en la Patagonia Austral, 1880-1920. Universidad Federal de la Patagonia Austral. Río Gallegos, Santa Cruz. 475 pp.

BARNHURST, D. 1986. Vulnerability of cougars to hunting. Thesis, Utah State University. Logan, USA.

BARTEL, RA \& MW BRUNSON. 2003. Effects of Utah's coyote bounty program on harvester behavior. Wildl. Soc. Bull., 31:1-8.

Beausoleil, RA \& DA Martorello (eds.). 2005. Proceedings of the Eighth Mountain Lion Workshop. Olympia, Washington, USA. Pp. 229.

BERGER, KM. 2006. Carnivore-livestock conflicts: effects of subsidized predator control and economic correlates on the sheep industry. Conserv. Biol., 20:751-761.

Bibiloni, H. 2004. Documento Proyecto PNUD-FMAM. Gobierno de la República Argentina. Programa de Naciones Unidas para el Desarrollo. Fondo para el Medio Ambiente Mundial. Manejo Sustentable de Ecosistemas Semi-Áridos para el Control de la desertificación de la Patagonia.

BJORGE, RR \& JR GUNSON. 1985. Evaluation of wolf control to reduce cattle predation in Alberta. J. Range Manage., 38:483-487.

Corbelle Rico, EJ \& E Rico Boquete. 2008. La actividad de 
las juntas de extinción de animales dañinos en España, 1944-1968. IX Congreso de la Asociación de Historia Contemporánea. Murcia.

Cougar Management Guidelines Working Group. 2007. Cougar management guidelines. 1st ed. Wildfutures Press, Bainbridge Island, Washington.

Crespo, EA; ACM Schiavini; G Perez Macri; L Reyes \& S DANS. 1994. Estudios para la determinación de la edad en mamíferos marinos del Atlántico Sudoccidental. Anales de la 4ta. Reunión de Trabajo de Especialistas en Mamíferos Acuáticos de América del Sur. Universidad Austral de Chile. Pp:31-55.

CURRIER, MJP. 1983. Felis concolor. Mamm. Species, 200:1-7.

Elbroch, ML \& HU WitTmer. 2013. The effects of puma prey selection and specialization on less abundant prey in Patagonia. J. Mammal., 94:259-268.

García Brea, A; SC Zapata; DE Procopio; R Martínez Peck \& A Travaini. 2010. Evaluación del interés de productores ganaderos en el control selectivo y eficiente de predadores en la Patagonia Austral. Acta Zool. Mex. (n. s.), 26:303-321.

GAY, SW \& TL BEST. 1995. Geographic variation in sexual dimorphism of the puma (Puma concolor) in North and South America. Southwest. Nat., 42:148-159.

GAY, SW \& TL BEST. 1996a. Age-related variation in skulls of the puma (Puma concolor). J. Mammal., 77:191-198.

GAY, SW \& TL BEST. 1996b. Relationships between abiotic variables and geographic variation in skulls of pumas (Puma concolor: Mammalia, Felidae) in North and South America. Zool. J. Linn. Soc., 117:259-282.

Golluscio, RA; VA Deregibus \& JM Paruelo. 1998. Sustainability and range management in the Patagonia steppes. Ecol. Austral, 8:265-284.

Haines, AM; ME Tewes \& LL LaAck. 2005. Survival and sources of mortality in ocelots. J. Wildl. Manag., 69: 255-263.

Hall, SA \& JM PARUELO. 2006. Environmental controls on lambing rate in Patagonia (Argentina): a regional approach. Journal of Arid Environments, 64:713-735.

Hammill, J. 2007. Policy Issues Regarding Wolves in the Great Lakes Region. Session 5 En Transactions of the 72nd North American Wildlife and Natural Resources Conference. Portland, Oregon.

Harper, E K; WJ Paul; LD Mech \& S Weisberg. 2007. Effectiveness of lethal, directed wolf-depredation control in Minnesota. J. Wildl. Manag., 72:778-784.

Hone, J. 1994. Analysis of vertebrate pest control. Cambridge University Press. New York. 272 pp.

IrazoQui, H. 1981. Mortalidad perinatal en lanares. Producción Animal, 8:15-37.

Kamler, JF \& PS Gipson. 2000. Home range, habitat selection, and survival of bobcats, Lynx rufus, in a prairie ecosystem in Kansas. Canadian Field Naturalist, 114:388-394.

KLEveZAL, GA \& SE Kleinenberg. 1967. Age determination of mammals from annual layers in teeth and bones. Israel Program for Scientific Translations, 1-166.

KnopfF, KH; AA KnopfF; A Kortello \& MS Boyce. 2010. Cougar kill rate and prey composition in a multiprey system. J. Wildl. Manag., 74:1435-1447.

KNOWLton, FE; EM GeSe \& MM JAeger. 1999. Coyote depredation control: an interface between biology and management. J. Range Manage., 52:398-412.

Logan, KA; LL IRwin \& R SkinNer. 1986. Characteristics of a hunted mountain lion population in Wyoming. J. Wildl.
Manag., 50:648-654.

Logan, KA \& LL SweAnor. 2001. Desert puma: evolutionary ecology and conservation of an enduring carnivore. Island Press. Washington, D.C., USA. 451 pp.

Montanelli, SB. 2008. Análisis del conflicto de prelación de puma sobre ganado ovino: Visión de los productores y actualización de instrumentos legales. Informe Técnico de la Dirección de Fauna y Flora Silvestre. Provincia del Chubut. $21 \mathrm{pp}$.

Omand, DN. 1950. The bounty system in Ontario. J. Wildl. Manag., 14:425-434.

ReYNolds, JC; HN Goddard \& MH BrockLess. 1993. The impact of local fox (Vulpes vulpes) removal on fox populations at two sites in southern England. Gibier Faune Sauvage, 10: 319-334.

RobinetTe, WL; JS GAShwiLER \& OW Morris. 1961. Notes on cougar productivity and life history. J. Mammal., 42:204-217.

Robinson, HS; RB Wielgus; HS Cooley \& SW Cooley. 2008. Sink populations in carnivore management: cougar demography and immigration in a hunted population. Ecol. Appl., 18:1028-1037.

Ross, PI \& MG JALKotzy. 1992. Characteristics of a hunted population of cougars in Southwestern Alberta. J. Wildl. Manag., 56:417-426.

Ruth, TK. 2004. Ghost of the Rockies: the Yellowstone cougar project. Yellowstone Sci., 12:13-24.

Seidensticker, JC IV; MG Hornocker; WV WiLes \& JP Messick. 1973. Mountain lion social organization in the Idaho Primitive Area. Wildl. Monogr., 35:3-60.

Stoner, DC; ML Wolfe \& DM Chonte. 2006. Cougar exploitation levels in Utah: implications for demographic structure, population recovery, and metapopulation dynamics. J. Wildl. Manag., 70:1588-1600.

Storm, GL; RD Andrews; RL Phillips; RA Bishop; DB SinifF \& JR TESTER. 1976. Morphology, reproduction, dispersal, and mortality of Midwestern red fox populations. Wildl. Manag., 49:3-82.

SUMINSKI, HR. 1982. Mountain lion predation on domestic livestock in Nevada. Proceedings of the Tenth Vertebrate Pest Conference. Paper 43. http://digitalcommons.unl.edu/ vpc10/43.

SUNQUIST, ME \& F SUNQUIST. 2002. Wild cats of the World. The University of Chicago Press. Chicago and London. 462 pp.

Travaini, A; SC Zapata; R Martínez-Peck \& M Delibes. 2000. Percepción y actitud humanas hacia la predación de ganado ovino por el zorro colorado (Pseudalopex culpaeus) en Santa Cruz, Patagonia Argentina. Mastozoología Neotropical, 7:117-129.

Treves, A \& U Karanth. 2003. Human-Carnivore conflict and perspectives on carnivore management worldwide. Conserv. Biol., 17:1491-1499.

Wainwright, CJ; CT DarimOnt \& PC PAQUET. 2010. British Columbia's Neglected Carnivore: a Conservation Assessment and Conservation Planning Guide for Cougars. Version 01. Raincoast Conservation Foundation. Sidney, BC. 58 pp.

Walker, S \& A Novaro. 2010. The World's Southernmost Pumas in Patagonia and the Southern Andes. Part II, Chapter 7, Pp. 91-99 en: M Hornocker y S Negri (eds). Cougar, Ecology and Conservation. University of Chicago Press. Chicago, USA. 304 pp.

West, GA. 1962. Wolf Control in British Columbia, Canada. Proceedings of the $1^{\text {st }}$ Vertebrate Pest Conference. Paper 6. http:/ /digitalcommons.unl.edu/vpcone/6.

WoODROFFe, R \& LG Frank. 2005. Lethal control of African lions (Panthera leo): local and regional population impacts. Anim. Conserv., 8:91-98. 